\title{
Relation between histological findings on early repeat right ventricular biopsy and ventricular function in patients with myocarditis ${ }^{\star}$
}

\author{
G WILLIAM DEC JR, $†$ JOHN T FALLON, † JAMES F SOUTHERN, \\ IGOR F PALACIOS $\uparrow$ \\ From the Cardiac Unit, $\uparrow$ Medical Services, and the $\ddagger$ Pathology Department, Massachusetts General Hospital \\ and Harvard Medical School, Boston, Massachusetts, USA
}

SUMMARY Histological findings on repeat endomyocardial biopsy and changes in left ventricular $\stackrel{+}{\dot{r} \omega}$ ejection fraction early during immunosuppressive treatment were studied in 20 patients with documented myocarditis. All patients presented with heart failure of less than six months' duration? and a left ventricular ejection fraction of $\leqslant 0.40$. Repeat biopsy and assessment of ventricular function were performed at a mean (SEM) of 79 (17) days after the initial biopsy. At repeat biopsy용 eight patients had evidence of ongoing myocarditis and 12 showed resolved myocarditis. In eighto $(66 \%)$ of the 12 patients with resolved myocarditis ventricular function had improved significantly. Left ventricular ejection fraction also improved significantly in four of eight patients during $\overrightarrow{0}$ treatment despite ongoing myocardial inflammation. Regardless of the histological findings on $\infty$ repeat biopsy, early improvement in ejection fraction was associated with an excellent long term prognosis-that is $83 \%$ survived for at least three years.

Histological resolution of myocarditis during immunosuppressive treatment is not a prerequisite⿳亠二口犬 for improvement in ventricular function; and changes in left ventricular ejection fraction during the first three months of treatment are predictive of clinical outcome.

Myocarditis can present suddenly as dilated cardiomyopathy. ${ }^{12}$ Examination of a biopsy specimen of the right ventricular endomyocardium can confirm myocarditis. Although the natural history and treatment of myocarditis remain uncertain, ${ }^{1-3}$ observations by Mason et al and Daley et al suggest that a subset of patients may show both histological and haemodynamic improvement as a result of immunosuppressive treatment. ${ }^{45}$ Serial biopsy of the right ventricular endomyocardium is often performed to assess the effect of treatment on myocardial inflammation and guide further management. The relation between histological findings and ventricular function during treatment has not been established.

We examined the relation between the histological appearance of the myocardium on repeat endomyocardial biopsy, changes in left ventricular function, and long term clinical outcome in a group of

Requests for reprints to Dr G William Dec, Jr, Cardiac Unit, Bulfinch One, Massachusetts General Hospital, Boston, MA 02114, USA.

* Presented in part at the 55th annual meeting of the American Heart Association, Washington, DC, November 1985.

Accepted for publication 19 May 1988 patients with symptomatic heart failure caused by myocarditis.

\section{Patients and methods}

\section{PATIENTS}

From a review of the medical and pathology records of 637 patients who had undergone right ventricular endomyocardial biopsy between 1 January 1980 ando 30 October 1986, we identified 20 consecutive $\triangle$ patients who met the following criteria: (a) symp-o tomatic heart failure lasting $\leqslant 6$ months; $(b)$ a left ventricular ejection fraction $\leqslant 0.40$ by radioven- $-\infty$ triculogram; (c) histological confirmation of myocar- $N$ ditis on initial right ventricular biopsy; $(d)$ repeat ${ }_{\mathcal{E}}^{N}$ biopsy and assessment of left ventricular functiono by radioventriculogram after the start of immuno-e suppressive treatment.

In all cases, the clinician had requested the initial? right ventricular biopsy for diagnostic purposes; the $D$ timing of biopsy relative to the duration of the patient's illness was determined only by the timing of this referral. Coronary angiography, performed in all $\underset{2}{2}$ patients, showed no significant obstructive lesions. 
TECHNIQUE OF TRANSVENOUS

ENDOMYOCARDIAL BIOPSY AND PATHOLOGICAL EXAMINATION

Right heart catheterisation was performed in all patients on at least two occasions with a triple lumen thermodilution catheter. A biopsy specimen of the endocardium was obtained after the haemodynamic measurements. We used a technique that resembled earlier descriptions. ${ }^{26}$ Multiple biopsy samples (usually 4-7, each measuring $1-2 \mathrm{~mm}$ in diameter) were taken from the right ventricular septum by means of a Caves-Schultz-Stanford bioptome inserted through the right internal jugular vein. Biopsy samples were processed according to standard staining and embedding methods. ${ }^{2}$

Biopsy specimens were classified on the basis of a review of all light microscopic slides, including plastic thick sections and frozen section tissue slides. Myocarditis was diagnosed on initial biopsy if there was a lymphocytic infiltrate, either focal or diffuse, in association with necrotic or degenerating myocytes. ${ }^{7}$ All repeat biopsy specimens were compared with that individual's initial biopsy specimen and were then classified according to the Dallas criteria ${ }^{7}$ into one of three categories:

(a) Ongoing myocarditis.-Specimens continued to show evidence of a persistent inflammatory infiltrate and ongoing degeneration or necrosis of adjacent myocytes.

(b) Resolving myocarditis.- Specimens showed continued cellular inflammation but no evidence of myocyte necrosis. The inflammatory infiltrate, although present, had decreased in amount.

(c) Resolved myocarditis. - These biopsy specimens showed resolution of both infiltrate and myocyte necrosis.

Each biopsy specimen was also graded for the degree of fibrosis according to the following semiquantitative scale: 0 , no fibrosis; $1+$, focal fibrosis; $2+$, multifocal interstitial fibrosis; and $3+$, diffuse interstitial or replacement fibrosis. The patients were then divided into two groups on the basis of histological findings on repeat biopsy: ongoing: myocarditis (including resolving histology) and resolved myocarditis.

\section{ASSESSMENT OF LEFT VENTRICULAR FUNCTION AND CLINICAL STATE}

At initial and follow up assessment the left ventricular ejection fraction was measured by multiple gated equilibrium radioventriculography. ${ }^{8}$ Right ventricular ejection fraction was measured in one patient with isolated right heart failure who had normal left ventricular function by first pass radioventriculogram. All ventriculograms were obtained within 14 days of the repeat right ven- tricular biopsy and most were performed the same day. Left ventricular function was considered to have improved during treatment if the left ventricular ejection fraction on the repeat ventriculogram had increased by $>0 \cdot 10$.

We also reviewed the medical records and questioned each patient about the severity of their symptoms and functional limitations caused by congestive heart failure. On the basis of information, each patient was assigned a New York Heart Association classification at the time of initial presentation, follow up biopsy, and most recent clinical evaluation. Clinical improvement was defined as: (a) a decrease in symptomatic heart failure by one or more New York Heart Association classes in association with $(b)$ a rise in ventricular ejection fraction by $\geqslant 0 \cdot 10$.

\section{TREATMENT PROTOCOLS}

All patients presented with symptomatic heart failure and had been receiving digoxin and diuretic treatment at the time of initial biopsy. Most patients were also receiving vasodilators (captopril or prazosin). Patients were given optimal medical treatment for heart failure before the initial measurement of ventricular function and treatment was not increased between the initial and follow up studies. Systemic anticoagulation and antiarrhythmic agents were used according to standard clinical indications.

All 20 patients were given immunosuppressive agents and underwent repeat biopsy during treatment as part of our routine treatment protocol. Prednisone was administered at an initial dose of 1.25 $\mathrm{mg} / \mathrm{kg} /$ day for the first week. The dose was then tapered to $0.33 \mathrm{mg} / \mathrm{kg} /$ day by the end of the twelfth week. The dosage remained constant for an additional eight weeks and was then tapered to zero by the end of week 24 . One patient did not consent to treatment with azathioprine. The remaining 19 patients were given azathioprine at a dose of $2.0 \mathrm{mg} /$ $\mathrm{kg}$ /day for the entire six months of treatment, unless leucopenia (white blood cell count $<5000 / \mathrm{ml}$ ) developed.

\section{STATISTICAL ANALYSIS}

Statistical analyses were performed by RS/1 (Bolt Beranek and Newman) COMPARE. A p value of $<0.05$ was regarded as statistically significant for a single set of data. All group data were expressed as the mean (SEM).

\section{Results}

The table shows the radioventriculographic, clinical, and histological characteristics of the 20 patients (mean age 45.8 (3.1) years) with myocarditis. Biventricular heart failure was present in all but one 
patient, who had predominantly right ventricular dysfunction. The mean initial group ejection fraction was $0.22(0.02)$ (range $0.07-0.40$ ) and New York Heart Association class was $3.7(0 \cdot 1)$. Repeat right ventricular biopsy was performed 79 (17) days (range 20-338) from initial sampling. The mean ventricular ejection fraction at the time of follow up examination had increased to $0.35(0.03)$ (range $0.18-0.58)(p=$ 0.0005 ) and the New York Heart Association class had decreased to $2 \cdot 0(0 \cdot 2)(p \leqslant 0.01)$.

\section{HISTOLOGICAL FINDINGS AND VENTRICULAR FUNCTION}

The table shows the changes that we saw in left ventricular function during immunosuppressive treatment in each patient. Doses of diuretic and vasodilators were either unaltered or reduced between the initial and follow up assessments of ventricular function (data not shown). The individual response to immunosuppressive treatment was highly variable. Left ventricular function improved in $12(60 \%)$ patients and remained unchanged or declined in eight patients. Figure 1 shows the change in ventricular ejection fraction for each patient by histological classification on repeat biopsy. Eight patients had ongoing myocarditis and in 12 patients histological examination showed resolved myocarditis. The timing of follow up biopsy and radioventriculographic measurement of ventricular function did not differ between the two histological groups: group with ongoing myocarditis 86 (21) days $v$ group with resolved myocarditis 75
Dec, Fallon, Southern, Palacio

(25) days $(p>0.05)$. Improvement in systolic ventricular performance was independent of myocardial inflammation on repeat biopsy an $\overline{\bar{s}}$ occurred in four of eight patients with ongoing myocarditis compared with eight of 12 (66\% patients with resolved myocarditis. The mean ejec $\frac{\bar{s}}{}$. tion fraction for the group with resolved myocarditi increased from $0.20(0.02)$ to $0.35(0.05)(p=0.001$ d while that of the group with ongoing myocarditis rose from $0.25(0.03)$ to $0.34(0.04)(p=0.03)$.

The degree of fibrosis seen on initial and follow up endomyocardial biopsy was also measured and analysed to determine its effect on changes in ven tricular function. Initial biopsy showed no fibrosis irg seven (35\%) patients, focal or multifocal fibrosis in nine $(45 \%)$, and diffuse interstitial or replacementw fibrosis in the remaining four patients. The fibrosis scores in the groups with ongoing and resolved myocarditis were $1.4(0.4)$ and $1.2(0.3)$ respectively at initial biopsy and $1.4(0.5) v 1.0(0.3)$ at follow up biopsy. The extent of fibrosis on initial or repeat biopsy did not correlate with changes in ejection fraction.

CLINICAL IMPROVEMENT AND OUTCOME Clinical improvement (a decrease in New York Hear' Association symptom class and a rise in ventriculap ejection fraction) was a common finding during the treatment for myocarditis. Improvement was seen in seven $(58 \%)$ of 12 of the patients with resolveco myocarditis and in four of eight with ongoing myocarditis ( $p=N S$ ). Not surprisingly, an improvement in symptoms was more common when

Table Histological, haemodynamic, and clinical characteristics of 20 patients with treated myocarditis

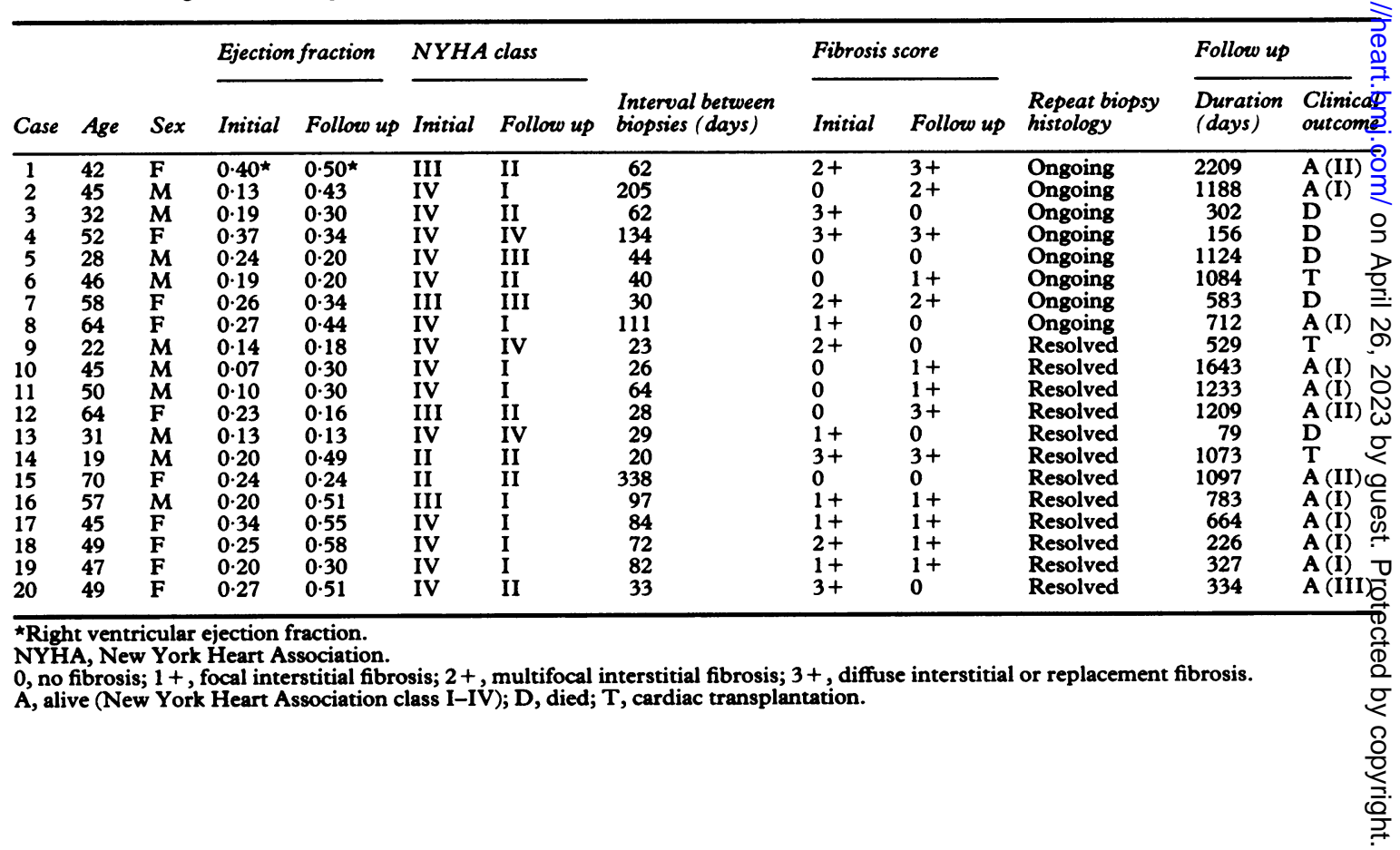



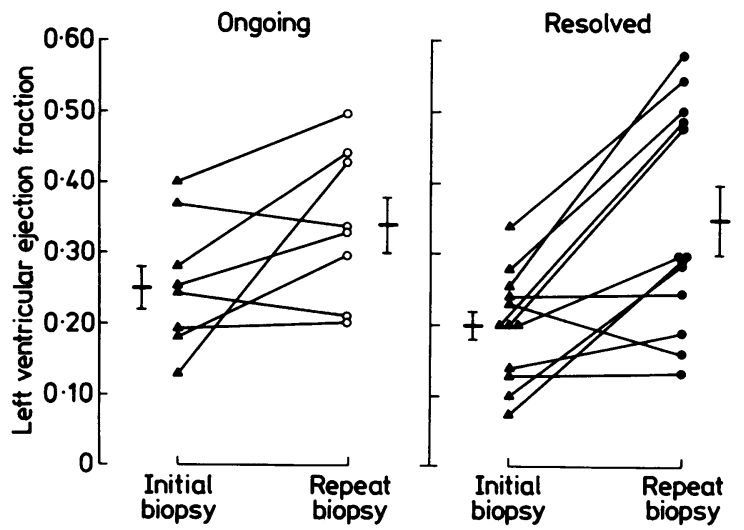

Fig 1 Left ventricular ejection fraction for each patient at initial and repeat biopsy. Patients are grouped according to histological findings on repeat biopsy.

the ventricular ejection fraction increased. Eleven of the 12 patients who showed a substantial increase in ejection fraction had a decline in New York Heart Association class. The one patient (case 14) whose symptoms did not improve despite an increase in left ventricular systolic performance had only mild (class II) symptoms at the time of both initial and follow up evaluations.

Clinical improvement after completion of six months of immunosuppressive treatment was highly dependent upon changes in ventricular function rather than on histological findings on repeat endomyocardial biopsy. In 12 patients the ejection fraction improved during treatment from $0.22(0.03)$ to $0.43(0.03) ; p<0.05)$ and all were alive one year after initial biopsy. Repeat biopsy showed resolved myocarditis in eight patients and ongoing myocarditis in the remaining four. Fibrosis was either absent or focal in nine of these 12 patients and the fibrosis score for the group was $1.2(1.0)$. Although serial assessments of ventricular ejection fraction and myocardial histology were not available for every patient, complete clinical follow up was collected. The mean duration of clinical follow up for the group showing an improvement was 891 (175) days (range 226-2209 days). Ten patients are alive. In one patient left ventricular function gradually declined and he died of sepsis. Refractory myocarditis developed in the other individual who underwent cardiac transplantation. The mean New York Heart Association class for the group improved from a mean value of 3.7 $(0.2)$ at initial biopsy to $1.3(0.1)(p<0.05)$ at the time of repeat biopsy. This improvement in functional capacity was sustained after the end of immunosuppressive treatment, as shown by a mean New York Heart Association class of $1.7(0.3)$ at the time of most recent clinical evaluation (fig 2 ). Of the 10 surviving patients, eight remain in class $I$.

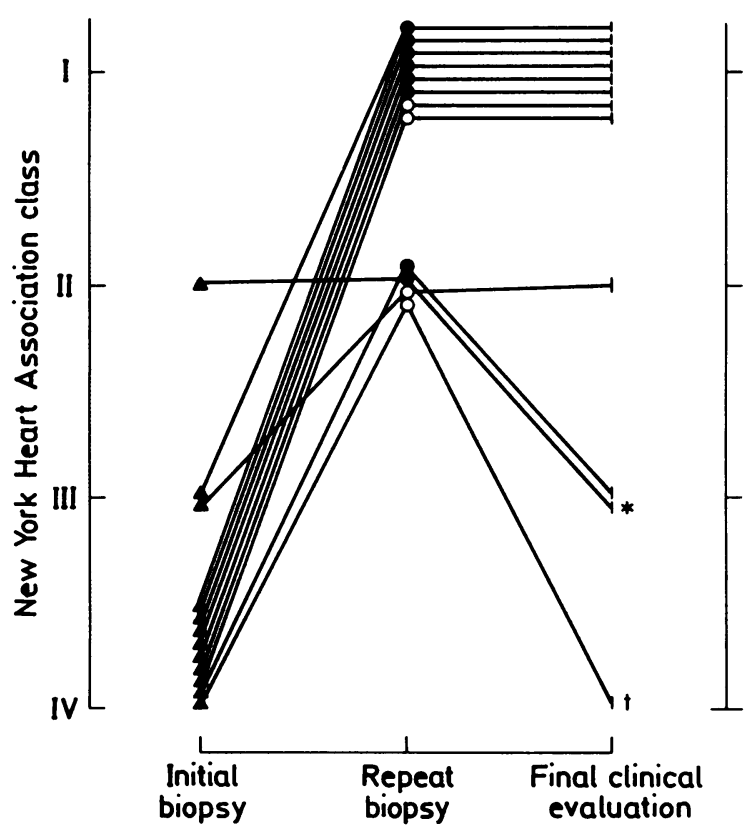

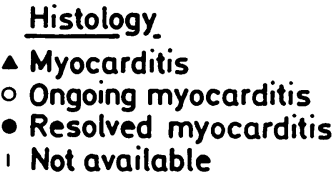
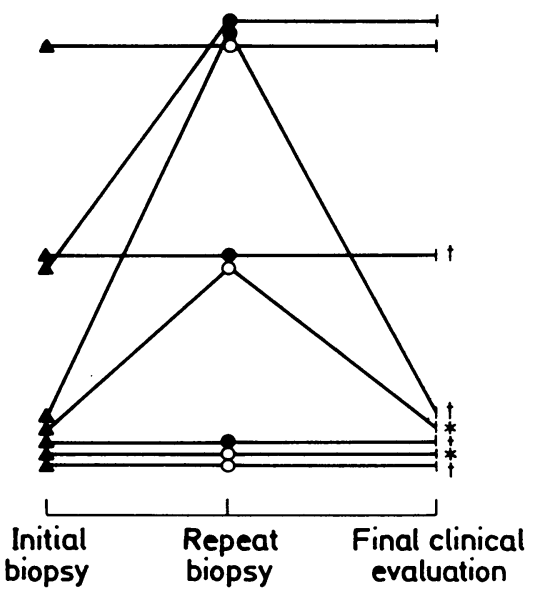

Fig 2 New York Heart Association classification for each patient at the time of initial biopsy, repeat biopsy, and final clinical assessment. Patients are grouped by improvement or lack of improvement in left ventricular ejection fraction (LVEF) at the time of repeat biopsy. Improvement was defined as an increase in $L V E F$ of $\geqslant 0 \cdot 10$. 
Ventricular function did not improve early during immunosuppressive treatment in eight patients $(0.22$ $(0.03)$ to $0.22(0.03))$. Clinical follow up for this group averaged 732 days (range 79-1209). Histological findings on repeat biopsy showed ongoing myocarditis in four patients and resolved myocarditis in the remaining four. Fibrosis on both initial and repeat biopsy was generally focal and fibrosis scores resembled those in the improved group. Two patients died of progressive heart failure in the first year after initial biopsy. Two additional patients died during the second year, while two others underwent cardiac transplantation for refractory heart failure. Only two non-transplanted patients are alive and both have considerable functional limitations (New York Heart Association class III). The mean class at the time of initial biopsy, repeat biopsy, and final determination either before death or transplantation or during most recent clinical evaluation was 3.5 $(0.03), 3.0(0.03$, and 3.4 (0.03) (p = NS) respectively (fig 2).

\section{Discussion}

Although endomyocardial biopsy has shown that myocarditis is associated with acute cardiac dilatation and symptomatic heart failure, ${ }^{9-11}$ both the prognosis and the most effective treatment remain uncertain. Uncontrolled observations ${ }^{4512}$ have suggested that immunosuppression may be of benefit in certain individuals, but no randomised clinical trial has yet been completed. In the absence of this information, a growing number of patients are now being treated with immunosuppressive agents once myocarditis has been histologically confirmed. The most effective method of monitoring the effects of treatment in patients with myocarditis is as controversial as the type of treatment used. Most centres that use immunosuppressive agents routinely perform repeat endomyocardial biopsy within 12-16 weeks of the start of treatment. Although immunosuppressive agents can reduce or eliminate lymphocytic inflammation and myocyte necrosis within several months, resolution can be spontaneous. ${ }^{13-15}$ Most $(60 \%)$ patients in our study showed resolved myocarditis at repeat biopsy after an average of 11 weeks of immunosuppressive treatment. None the less, these histological findings correlated poorly with changes in left ventricular ejection fraction and functional state. Left ventricular function improved and symptoms decreased in four of eight patients with histological evidence of ongoing myocarditis and in $60 \%$ of those individuals with resolved myocarditis.

It is not entirely surprising that histological and haemodynamic improvements do not parallel one another. There are several explanations for this apparent discrepancy. One possibility is that the transvenous biopsy technique does not provide a representative histological picture of either the degree of ongoing inflammation or interstitial fibrosis in a disease that is often focal or multifocal. ${ }^{16}$ Many patients who seemed to have resolved myocarditis may have had other areas of myocardial inflammation and myocyte necrosis that were undetected on repeat biopsy. It is also possible that those patients who did not improve had a greater degree of fibrosis and irreversible ventricular dysfunction before the start of treatment. This explanation seems unlikely since both the initial fibrosis score and ejection fraction were similar in the two groups. Another explanation is that the histological findings obtained from right ventricular biopsy do not reflect the events that occur in the left ventricle during myocarditis, where the haemodynamic dysfunction has most effect. This explanation remains possible, although unlikely, because previous pathological studies have confirmed that right ventricular sampling is representative of left ventricular histological findings in most cases of myocarditis. ${ }^{17}$

Another possibility is that those patients with ongoing myocarditis presented earlier in their illness and were treated before the development of permanent left ventricular dysfunction. While all patients reported heart failure symptoms lasting $<6$ months, and the interval between the development of symptoms of heart failure and initial right ventricular biopsy did not appear to be shorter in the group with ongoing myocarditis, this possibility cannot be excluded because the duration of illness was based upon the patient's subjective estimation of the onset of symptoms.

\section{CLINICAL IMPROVEMENT AND OUTCOME}

During treatment with prednisone alone or with azathioprine both ventricular function and clinical symptoms improved in approximately $60 \%$ of individuals with symptomatic heart failure of recent onset caused by myocarditis. These findings confirm previous biopsy studies ${ }^{41214}$ and uncontrolled observations in a larger series of our own patient population and suggest that a subset of patients may improve as the result of immunosuppression.

While no relation was seen between changes in ventricular function during treatment and the presence or absence of myocardial inflammation on repeat endomyocardial biopsy, a substantial rise in ejection fraction within the first three months of immunosuppressive treatment was predictive of sustained improvement in functional state. Ten of the 12 patients who showed early improvement in ventricular function are alive, and all but two are in New York Heart Association class I. This contrasts with the eight patients who did not show an early improvement in ejection fraction during treatment. 
Six of them have died or undergone cardiac transplantation for progressive heart failure, while the remaining two patients continue to experience considerable functional limitations. While several patients who improved did eventually show clinical deterioration and worsening symptoms during long term follow up, perhaps because of reactivation of myocarditis, patients who did not show an improvement in symptoms and functional class at the time of repeat biopsy and clinical assessment did not do so later.

These findings are similar to a smaller series recently reported by Quigley et al which showed that an improvement in ventricular function within 6-8 months of immunosuppressive treatment for myocarditis was highly predictive of an excellent long-term prognosis. ${ }^{18}$ Further, their study also supports our observation that neither the presence of myocardial inflammation nor the degree of fibrosis on repeat biopsy was predictive of functional improvement. The present study suggests that patients with an excellent prognosis can be identified by changes in left ventricular ejection fraction as early as three months after the start of treatment.

But not all patients who improved during this study did so as a direct result of immunosuppressive treatment. Spontaneous improvement in ventricular function has already been reported. ${ }^{14}$ At present those patients who will experience spontaneous symptomatic and haemodynamic improvement cannot be distinguished from those in whom immunosuppressive treatment may be of substantial benefit. We did not attempt to determine the role of immunosuppression in the treatment of myocarditis and there was no randomised control group in our study.

We examined only the relation of histological findings during treatment and changes in ventricular function early during the course of immunosuppressive treatment. Therefore, although clinical outcome and functional class were followed over many months, we did not obtain serial biopsy specimens or measure ejection fraction after the end of immunosuppressive treatment. We do not know whether continued myocardial inflammation is compatible with a sustained improvement in ventricular performance over the course of many months or years, though this seems unlikely. Finally, it does not seem prudent to recommend alteration in immunosuppressive treatment early during the course of treatment on the basis of the presence or absence of ongoing myocardial inflammation on repeat biopsy.

\section{References}

1 Abelmann WM. Viral myocarditis and its sequellae. Annu Rev Med 1973;24:145-52.

2 Parillo JE, Aretz HT, Palacios I, Fallon JT, Block PC. The results of transvenous endomyocardial biopsy can frequently be used to diagnose myocardial diseases in patients with idiopathic heart failure: endomyocardial biopsies in 100 consecutive patients revealed a substantial incidence of myocarditis. Circulation 1984; 69:93-101.

3 Zee Cheng CS, Tsai CC, Palmer DC, Codd JE, Pennington DC, Williams GA. High incidence of myocarditis by endomyocardial biopsy in patients with idiopathic congestive cardiomyopathy. J Am Coll Cardiol 1984;3:63-70.

4 Mason JW, Billingham ME, Ricci DR. Treatment of acute inflammatory myocarditis assisted by endomyocardial biopsy. Am J Cardiol 1980;45: $1037-44$.

5 Daly K, Richardson PJ, Olsen EGJ, et al. Acute myocarditis: role of histological and virological examination in the diagnosis and assessment of immunosuppressive treatment. $\mathrm{Br}$ Heart $J$ 1984; 51:30-5.

6 Mason JW. Techniques for right and left ventricular endomyocardial biopsy. Am J Cardiol 1978;41: 887-92.

7 Aretz HT, Billingham ME, Edwards WD, et al. Myocarditis: a histopathologic definition and classification. Am J Cardiovasc Pathol 1986;1:3-14.

8 Burrow RD, Strauss MW, Singleton R, et al. Analysis of left ventricular function in multiple gated acquisition cardiac blood pool imaging: comparison to contrast angiography. Circulation 1977;56:1024-8.

9 Lerner AM, Wilson FM, Reys MP. Enteroviruses and the heart (with special emphasis on the probable role of coxsackie virus, group B, types 1-5) in epidemiological and experimental studies. Mod Concepts Cardiovasc Dis 1975;44:7-10.

10 Smith WG. Coxsackie B myopericarditis in adults. $\mathrm{Am}$ Heart J 1970;80:34-46.

11 Sainani GS, Krompotic E, Slodki SJ. Adult heart disease due to coxsackie virus $\mathrm{B}$ infection. Medicine (Baltimore) 1968;47:133-47.

12 O'Connell JB, Robinson JA, Henkin PE, Gunnar RM. Immunosuppressive therapy in patients with congestive cardiomyopathy and myocardial uptake of Gallium-67. Circulation 1984;64:780-6.

13 Fenoglio JJ Jr, Ursell PC, Kellogg CF, Drusin RE, Weiss MB. Diagnosis and classification of myocarditis by endomyocardial biopsy. N Engl J Med 1983; 308:12-8.

14 Dec GW Jr, Palacios IF, Fallon JT, et al. Active myocarditis in the spectrum of acute dilated cardiomyopathies: clinical features, histologic correlates, and clinical outcome. $N$ Engl J Med 1985;312:885-90.

15 Hosenpud JD, McAnulty JM, Niles NR. Lack of objective improvement in ventricular systolic function in patients treated with azathioprine and prednisone. J Am Coll Cardiol 1985;6:797-801.

16 Miklozek CL, Parker JA, Royal HD, Come PC, Modlin JF, Abelmann WH. Radionuclide ventriculography in viral myocarditis patients [Abstract]. Circulation 1980;62(suppl III):318.

17 Weiland DS, Donaldson RF, Isner JF. How well does the endomyocardial biopsy represent the state of the heart? [Abstract]. Circulation 1984;107(suppl II):401.

18 Quigley PJ, Richardson PJ, Meary BT, et al. Long term follow-up of acute myocarditis. Correlation of ventricular function and outcome. Eur Heart $J$ 1987; 8(suppl J):39-42. 